\title{
Identification of the Most Accessible Sites to Ribozymes on the Hepatitis C Virus Internal Ribosome Entry Site
}

\author{
Kyung-Ju Ryu and Seong-Wook Lee* \\ Department of Molecular Biology, Dankook University, Seoul 140-714, Korea
}

Received 14 April 2003, Accepted 30 May 2003

\begin{abstract}
The hepatitis $\mathrm{C}$ virus (HCV) is a major causative agent of chronic hepatitis and hepatocellular carcinoma. The development of alternative antiviral therapies is warranted because current treatments for the HCV infection affect only a limited number of patients and lead to significant toxicities. The HCV genome is exclusively present in the RNA form; therefore, ribozyme strategies to target certain HCV sequences have been proposed as anti-HCV treatments. In this study, we determined which regions of the internal ribosome entry site (IRES) of $\mathrm{HCV}$ are accessible to ribozymes by employing an RNA mapping strategy that is based on a trans-splicing ribozyme library. We then discovered that the loop regions of the domain IIIb of HCV IRES appeared to be particularly accessible. Moreover, to verify if the target sites that were predicted to be accessible are truly the most accessible, we assessed the ribozyme activities by comparing not only the transsplicing activities in vitro but also the trans-cleavage activities in cells of several ribozymes that targeted different sites. The ribozyme that could target the most accessible site identified by mapping studies was then the most active with high fidelity in cells as well as in vitro. These results demonstrate that the RNA mapping strategy represents an effective method to determine the accessible regions of target RNAs and have important implications for the development of various antiviral therapies which are based on RNA such as ribozyme, antisense, or siRNA.
\end{abstract}

Keywords: Gene therapy, HCV, IRES, RNA mapping, Transsplicing ribozyme

\section{Introduction}

The hepatitis $\mathrm{C}$ virus (HCV) infection causes chronic liver diseases that can frequently lead to hepatocellular carcinoma (Lauer and Walker, 2001). Worldwide, there are about 170 million infected individuals (World Health Organization, 1999). Presently, the only treatments for the HCV infection are $\alpha$-interferon $(\alpha$-IFN) or, more recently, a polyethylene glycol modified form of $\alpha$-IFN in combination with rivabrin (Bisceglie et al., 2002). With these treatments, however, many chronically ill patients fail to sustain virological benefits and show significant toxicities (Manns et al., 2001; Fried et al., 2002). Therefore, more improved therapeutic modalities are needed for the HCV infection.

$\mathrm{HCV}$ is a member of the human flavivirus family with a positive-stranded 9,600-nucleotide(nt) RNA genome (Miller and Purcell, 1990; Choo et al., 1991). Since the viral genome is exclusively present in the RNA form, then several ribozyme- (Lieber et al., 1996; Sakamoto et al., 1996; Welch et al., 1996; Macejak et al., 2000), antisense oligonucleotide(Hanecak et al., 1996; Witherell, 2001), or siRNA-based strategies (Kapadia et al., 2003; Randall et al., 2003; Wilson et al., 2003) to specifically target certain HCV sequences have been proposed as HCV therapeutics. However, one key factor to successfully apply these RNA-based anti-HCV treatments to clinical settings is to find the most accessible sites in the target RNA sequences. This is because the target RNAs would form complex tertiary configurations and have proteins bound to them in cells (Lan et al., 2000).

The group I ribozyme from Tetrahymena thermophila was previously demonstrated to trans-splice an exon that is attached to its 3' end onto a separate 5' exon RNA, not only in vitro (Been and Cech, 1986) but also in E. coli (Sullenger and Cech, 1994) and mammalian cells (Jones et al., 1996). We, as well as other groups, have shown that these group I-based trans-splicing ribozymes can revise mutant transcripts that are associated with several human genetic and malignant diseases (Lan et al., 1998; Phylactou et al., 1998; Watanabe and Sullenger, 2000; Rogers et al., 2002; Shin et al., 2002).
*To whom correspondence should be addressed.

Tel: 82-2-709-2905; Fax: 82-2-798-4733

E-mail: SWL0208@unitel.co.kr 
Moreover, we demonstrated that the trans-splicing ribozymes could selectively and specifically induce therapeutic gene activities in HCV RNA-expressing cells (Ryu et al., 2003). The potential of the ribozymes to treat various human diseases is based on the ribozyme activity to specifically replace a target RNA with any RNA molecule to express. There is extremely malleable flexibility to select specific RNA molecules because virtually any $U$ residue in a 5' exon can be targeted for splicing by altering the nucleotide composition of the 5' exon binding site, called internal guide sequence (IGS), on the ribozyme to make it complementary to a target sequence that is present on the substrate RNA (Sullenger, 1996). In addition, the trans-splicing ribozyme generates reaction products that are stable in cellular circumstances, and hence easily detected by RT-PCR and readily assayed, providing experimental systems to optimize ribozyme catalysis in cells (Jones and Sullenger, 1997). Therefore, the trans-splicing ribozyme library with randomized IGS can be used for mapping the most accessible reacting sites to ribozymes on any target RNA.

In this study, we determined which regions of the HCV RNA sequence are most accessible to ribozymes by employing an RNA mapping strategy with the trans-splicing ribozyme library. The internal ribosome entry site (IRES), encompassing the 5'-UTR and N-terminal coding sequence of the core protein of HCV (Wang et al., 2000), was selected for targeting because the sequence is highly conserved among viral genotypes (Bukh et al., 1992) and critical for HCV replication (Rosenberg, 2001). In addition, we evaluated the mapping results by analyzing the activities of various ribozymes that target different sequences on HCV IRES in cells as well as in vitro.

\section{Materials and Methods}

Materials The restriction enzymes and reagents for RT-PCR and the in vitro transcription reaction were purchased from Roche Applied Science (Mannheim, Germany). Argininamide and most of the other chemicals were obtained from the Sigma Chemical Co. (St. Louis, USA). The DMEM tissue culture media and fetal bovine serum came from GIBCO (Grand Island, USA).

Mapping accessible sites on HCV IRES RNA To construct the mapping library (called $\mathrm{GN}_{5}$ library), IGS of the Tetrahymena group I trans-splicing ribozyme was randomized so that the 5' end of the ribozymes in the library began with 5'-GNNNNN-3', where $\mathrm{G}$ represents guanine and $\mathrm{N}$ represents equimolar amounts of the four nucleotides (nt) (Lan et al., 1998). Target RNA, HCV IRES (nts 18-402) of the HCV $1 \mathrm{~b}$ genomic RNA, was generated by in vitro transcription using T7 RNA polymerase with the BamHI fragment of $\mathrm{pH}(18-402)$ CAT (Hahm et al., 1998, a kind gift from $\mathrm{S}$. K. Jang, POSTECH University, Pohang, Korea). To map the HCV IRES RNA, $20 \mathrm{nM}$ of the $\mathrm{GN}_{5}$ library was incubated at $37^{\circ} \mathrm{C}$ for $3 \mathrm{~h}$ under splicing conditions (50 mM HEPES, $\mathrm{pH} 7.0,150 \mathrm{mM} \mathrm{NaCl}$, $\left.5 \mathrm{mM} \mathrm{MgCl}_{2}\right)$ in the presence of a guanosine $(100 \mu \mathrm{M})$ with $50 \mathrm{nM}$ of HCV IRES RNA. The reaction products were reverse- transcribed at $37^{\circ} \mathrm{C}$ for $30 \mathrm{~min}$ in the presence of argininamide $(10 \mathrm{mM})$ with a 3 ' tag primer specific for the 3' exon lac $Z$ sequence of the ribozyme (5'-ATGTGCTGCAAGGCGATT-3') (Jones et al., 1996). The cDNAs were then amplified by PCR for 35 cycles using the same 3' primer and a 5' primer I encompassing the 5' end of the target HCV IRES RNA (5'-GGGGAATTCGGGCGAATTGGGTA CCG G-3') or a 5' primer II specific for the sequence nt 148-165 of HCV IRES (5'-GGGGAATTCCTGCGGAACCGGTGAGTA-3'). The amplified trans-splicing products were cloned into a pUC19 vector and the inserts were then sequenced using the dideoxy termination method (Chung et al., 2002).

Ribozyme construction Specific ribozymes (such as Rib86-, Rib195-, Rib199-, Rib251-, Rib329-, or Rib380-3'tag that recognize the uridine at position $86,195,199,251,329$, or 380 , respectively, on the HCV IRES RNA) were generated by in vitro transcription of the DNA templates which were created from pT7L21 by PCR with a 5' primer that contained the T7 promoter and IGS of each ribozyme, as well as with a 3' primer specific for the 3' exon lacZ sequence. The pT7L-21 vector encoded a slightly shortened version of the natural group I intron from Tetrahymena, called L-21 (Sullenger and Cech, 1994). The IGS on the L-21 trans-splicing ribozyme (5'-GGAGGG-3') was exchanged with 5'GUGGCU-3' in Rib86-3'tag, 5'-GAGGAC in Rib195-3'tag, 5'GAGAAA-3' in Rib199-3'tag, 5'-GGCAGU-3' in Rib251-3'tag, 5'-GCGAGA-3' in Rib329-3'tag, or 5'-GCGUUU-3' in Rib3803 'tag. In addition, inactive ribozymes [R(d)86-, R(d)195-, R(d)199-, R(d)251-, R(d)329-, or R(d)380-3'tag, which lack part of the catalytic core of the enzyme (Sullenger and Cech, 1994)], were constructed as negative controls.

In vitro assay of ribozyme activity For the in vitro trans-splicing reaction assay of the ribozymes, the individual ribozymes with $3^{\prime}$ tag $(100 \mathrm{nM})$ were incubated at $37^{\circ} \mathrm{C}$ for $3 \mathrm{~h}$ under splicing conditions with HCV IRES RNA $(10 \mathrm{nM})$. The resulting RNA was reverse-transcribed at $37^{\circ} \mathrm{C}$ for $30 \mathrm{~min}$ in the presence of argininamide $(10 \mathrm{mM})$ with a 3 ' primer specific for the 3' exon lac $Z$ sequence of the ribozyme, as previously described. The cDNAs were then amplified by PCR for 35 cycles with the same 3' primer and with either a 5' primer I (specific for the 5' end of $\mathrm{HCV}$ IRES RNA) for the reaction with Rib86 or R(d)86, or a 5' primer II (specific for the sequence nt 148-165 of HCV IRES) for the reaction with the other ribozymes. The reaction products were analyzed on a 3\% agarose gel. The RT-PCR products were eluted from the gel, cloned onto a pUC19 vector, and then sequenced with the dideoxy termination method.

For the in vitro trans-cleavage reaction assay, each ribozyme without a 3'tag was produced by in vitro transcription of the ScaIdigested DNA fragments of the templates that were used for the generation of ribozymes with a 3 'tag. The ribozymes $(50 \mathrm{nM})$ were incubated at $37^{\circ} \mathrm{C}$ for $4 \mathrm{~h}$ under splicing conditions with $5^{\prime}$ end radio-labeled HCV IRES RNA (500 pM). The resulting products of the cleavage reactions were resolved on $5 \%$ acrylamide/7 $\mathrm{M}$ urea gel and analyzed by autoradiography.

Trans-cleavage reaction in cells The substrate RNA, IRES/FLuc RNA, which encodes HCV IRES followed by firefly luciferase (FLuc) RNA, was created by the in vitro transcription of the DNA 
templates that were amplified by PCR from the $\mathrm{pR} / \mathrm{HCV} / \mathrm{F}$ plasmid that is a dicistronic vector expressing renilla luciferase (RLuc) under the CMV promoter and firefly luciferase (FLuc) under the control of HCV IRES. This was kindly donated by S. K. Jang. RLuc RNA was generated by the in vitro transcription of the SalIdigested $\mathrm{pR} / \mathrm{HCV} / \mathrm{F}$ plasmid. The RNAs that were used for the transfection into cells were modified at its 3' end by the addition of poly(A) using poly(A) polymerase (Amersham Biosciences, Piscataway, USA) at $37^{\circ} \mathrm{C}$ for $20 \mathrm{~min}$. For the trans-cleavage reaction in the cells, the $293 \mathrm{~T}$ cells were plated at $3.0 \times 10^{5}$ cells per well in $35 \mathrm{~mm}$ dishes $24 \mathrm{~h}$ prior to transfection. The cells were cotransfected with $0.5 \mu \mathrm{g}$ IRES/FLuc RNA along with $0.5 \mu \mathrm{g}$ RLuc RNA and $4 \mu \mathrm{g}$ tRNA, active or inactive ribozymes using $3 \mu \mathrm{l}$ DMRIE-C (Invitrogen, Carlsbad, USA). The cell lysates were harvested $24 \mathrm{~h}$ after transfection, and the reporter gene activities were assessed by measuring relative light units using a luminometer TD-20/20 (Turner Designs Instrument) and dual-luciferase reporter assay system (Promega, Madison, USA) (Kim and Park, 2002).

\section{Results and Discussion}

Mapping of ribozyme-accessible uridines in HCV IRES RNA HCV IRES RNA can be recognized by the transsplicing ribozyme by base pairing to any accessible uridine residue in the RNA through IGS of the ribozyme. However, only a limited number of uridines on the target RNA can be actually accessible to the ribozyme, due to the substrate RNAs complex but stable tertiary structure (Lan et al., 2000). To determine which uridines in the HCV IRES RNA are accessible to ribozymes, an RNA mapping strategy was conducted. The mapping method was based on a transsplicing ribozyme library (Lan et al., 1998, 2000) and RNA tagging (Jones et al., 1996) (Fig. 1).

The ribozyme library, called the $\mathrm{GN}_{5}$ library (constructed based on the Tetrahymena group I intron), contains a randomized IGS. Thus, ribozymes in the $\mathrm{GN}_{5}$ library will react with and cleave to the substrate RNA at any accessible uridine $(\mathrm{U})$ residue and transfer a 3' exon to the 3' end of a 5' cleavage target product. Part of the lac $Z$ gene was employed as a 3' exon and molecular tag in the $\mathrm{GN}_{5}$ library that can be spliced onto the target RNA's Us that are accessible to the ribozyme. To map the HCV IRES RNA, the $\mathrm{GN}_{5}$ library was incubated under splicing conditions with the substrate $\mathrm{HCV}$ IRES RNA that is generated by in vitro transcription (nt 18402). The trans-splicing reaction products were amplified by RT-PCR. Two different amplification reactions were performed with a 3' primer (specific for the ribozyme's 3' exon lac $Z$ sequence) and two different 5 ' primers, 5' primer I (encompassing the 5' end of target HCV IRES RNA) or a 5' primer II (specific for the sequence nt 148-165 of HCV IRES), in order to exclude possibilities of missing any long amplified products from ribozyme reactions with the 3' part of the target RNA. The amplified products were then cloned and sequenced.

Sequence analyses of the splicing junction sites showed that

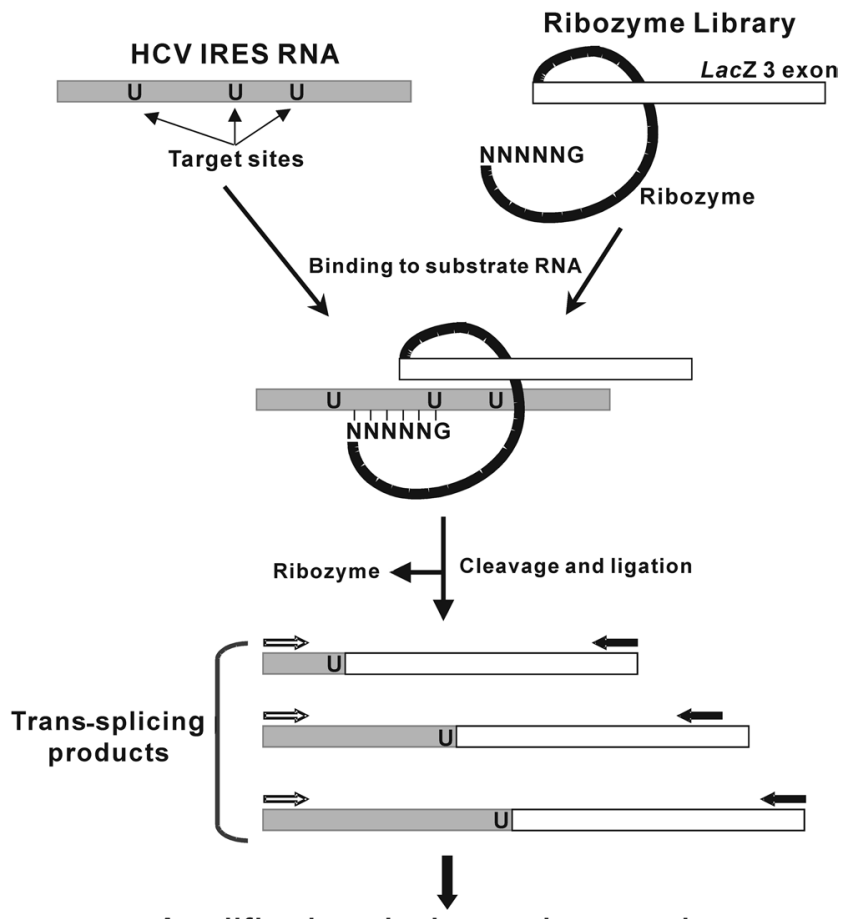

Amplification, cloning, and sequencing

Fig. 1. Scheme for mapping the accessible sites in HCV IRES RNA in vitro with a trans-splicing ribozyme library. The GN5 ribozyme library $(20 \mathrm{nM})$ and target $\mathrm{HCV}$ IRES transcript $(50 \mathrm{nM})$ were incubated in the reaction buffer at $37^{\circ} \mathrm{C}$ for $3 \mathrm{~h}$ in the presence of guanosine $(100 \mu \mathrm{M})$. The reaction products were amplified with a 5, primer, which was specific for HCV IRES RNA, and with a 3' primer that recognized the 3' exon tag lacZ sequence, cloned, and sequenced.

several uridines that are present in the loop regions of domain IIIb appeared to be particularly accessible, because almost $50 \%$ of the reaction products resulted from splicing at these sites (Fig. 2). Particularly, the most accessible site was present in the uridines at position 199 on the HCV IRES RNA.

Evaluation of RNA mapping studies To verify if the sites that were predicted to be accessible by the mapping studies were truly the most accessible sites to ribozymes, we assessed the trans-splicing activities of three different ribozymes that target uridines at positions 195 (U195), 199 (U199), or 251 (U251) in HCV IRES that were detected via a mapping analysis, or one ribozyme that targeted uridine at position 380 (U380) that was not identified from our mapping study (Fig. 3A). The Rib195-, Rib199-, Rib251-, or Rib380-3'tag ribozymes that recognized these individual sites were generated by in vitro transcription and incubated under splicing condition with the target HCV IRES RNA. RT-PCR analyses were then performed with a 3 ' primer that was specific for the 3' exon tag sequence and a 5' primer that was specific for HCV IRES, as previously described. An amplified fragment of the expected size of $87 \mathrm{bp}, 91 \mathrm{bp}$, or $143 \mathrm{bp}$ was generated from the reaction mixtures with the HCV IRES 


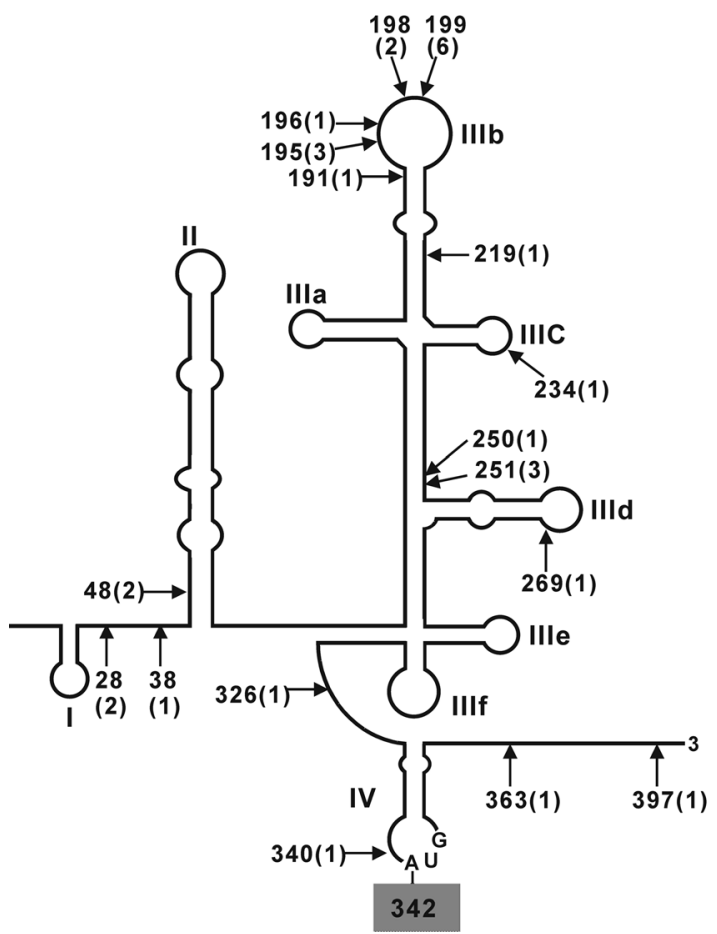

Fig. 2. Mapping results of the ribozyme-accessible sites in HCV IRES RNA. Nucleotide positions of the accessible uridines that were identified from the in vitro mapping analysis are indicated as nucleotide numbers by arrows on the predicted secondary structure of HCV 5'UTR and immediately downstream ORF (Honda et al., 1999). The number of clones that contain a given uridine at the splice site is presented in parentheses. The AUG residue that is located at nt 342-344 in domain IV denotes the initiator codon of the $\mathrm{HCV}$ core protein.

RNA and Rib195-, Rib199-, or Rib251-3'tag, respectively (Fig. 3A, lanes 3-5). It should be noted that the Rib199-3'tag can trans-splice a 3' exon tag onto HCV IRES with the highest efficiency. However, it was difficult to detect this RTPCR product from a sample with Rib380-3'tag (Fig. 3A, lane 6), indicating that U380 in HCV IRES was barely accessible to the ribozyme. These results, therefore, indicate that the relative trans-splicing efficiency at the chosen sites is the same as the predicted accessibility from our mapping analyses. Inactive versions of all four ribozymes that lack part of the catalytic core of the enzyme produced no RT-PCR products in the reaction with the target RNA (Fig. 3A, lanes 7-10). Moreover, no trans-splicing product was generated from either sample with the RNA substrate alone or the Rib1993 'tag alone (Fig. 3A, lanes 11, 12). These results suggest that the amplified cDNA products that are found in lanes 3-5 in Fig. 3A are the result of the catalytic activity of the ribozymes.

Once it was ascertained the specific ribozymes performed the trans-splicing reaction to transfer their 3' exon onto the target HCV IRES RNA, we then attempted to determine if the

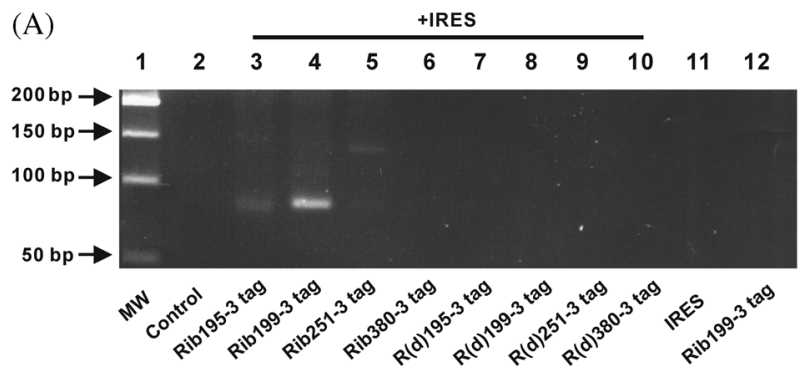

(B)
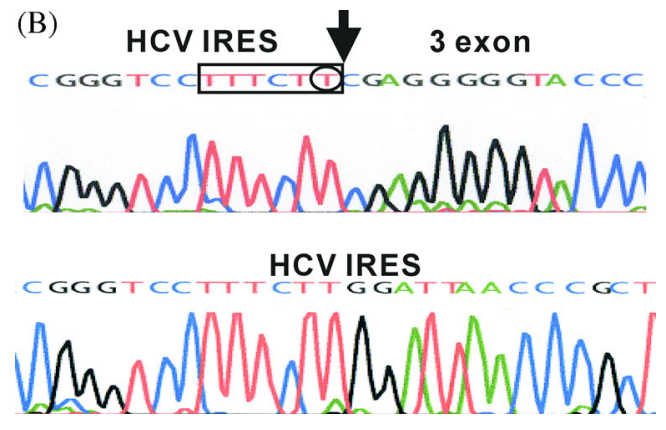

(C)
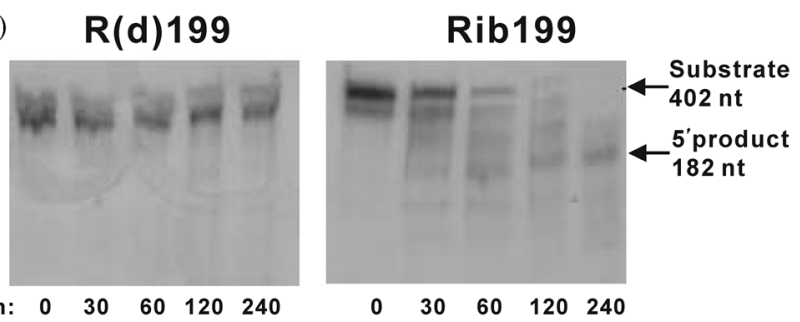

Fig. 3. In vitro trans-splicing and trans-cleavage activities. (A) RT-PCR analysis of trans-spliced RNA products generated in vitro. A series of active $(100 \mathrm{nM}$; lanes $3-6)$ or inactive ribozymes (100 nM; lanes 7-10) were incubated with the HCV IRES target RNA substrate $(10 \mathrm{nM})$, and the trans-spliced products were amplified. As a reaction control, the RT-PCR products without RNA (lane 2), with HCV IRES alone (lane 11), or Rib199 alone (lane 12) were presented. Amplification products were then subjected to electrophoresis in a $3 \%$ agarose gel. The migration of $50 \mathrm{bp}$ ladder is indicated as a molecular mass marker (lane 1, MW). (B) Sequence analysis of transsplicing products produced in vitro. The amplified products from the trans-splicing reaction between Rib199 and HCV IRES (Fig. $3 \mathrm{~A}$, lane 4) were isolated on a gel and cloned. Sequence of one representative clone (from 10 different clones with the same sequence) is shown. The expected sequence around the splicing junction, indicated by an arrow, is shown with the ribozyme recognition sequence in HCV IRES RNA boxed and the uridine at position 199 circled. The intact HCV IRES sequence is also represented in parallel. (C) In vitro cleavage of HCV IRES RNA. Active ribozyme, Rib199, or inactive ribozyme, R(d)199, (50 nM each) were incubated with the 5 ' end radiolabeled substrate HCV IRES RNA $(0.5 \mathrm{nM})$ and the aliquots were removed at $0,30,60$, 120, and $240 \mathrm{~min}$, as indicated. The cleavage reactions then were resolved on a $5 \%$ acrylamide/7 $\mathrm{M}$ urea gel. The uncleaved substrate and 5' cleavage products are indicated. 
ribozyme reaction could occur with fidelity. To this effect, sequence analyses of the spliced products (detected at lane 4 in Fig. 3A) were carried out. A sequence analysis of the 91-bp amplified fragment demonstrated that the ribozyme, Rib1993 'tag, had correctly reacted with HCV IRES at the predicted reaction site (U199) and replaced sequences downstream of the reaction sites with the 3' exon sequences tagged at the 3 ' end of the ribozyme (Fig. 3B). Sequencing of the reaction products that were isolated from lanes 3 or 5 of Fig. 3A demonstrated that the Rib195- or Rib251-3'tag also correctly spliced its 3' exon tag onto the HCV IRES target RNA at the predicted reaction site (data not shown). From these results, it was concluded that specific ribozymes that target the predicted accessible sites could react with high fidelity with the target RNA.

To confirm the activities of the ribozymes that target the sites that are predicted to be most accessible by mapping studies, the trans-cleavage activities of the ribozymes were next tested. To this effect, we constructed ribozymes without 3' exon (described in Materials and Methods) and incubated them with 5' end radio-labeled HCV IRES RNA. Rib199 without 3' exon were revealed to efficiently cleave a majority of the target HCV IRES RNA and yield products of the expected size in vitro (Fig. 3C). In contrast, no cleavage products of the target RNA were found with the inactive ribozyme $(\mathrm{R}(\mathrm{d}) 199)$, indicating that the cleavage products that were detected in the sample with Rib199 resulted from the catalytic activity of the ribozyme. Moreover, trans-cleavage activity Rib199 was the most efficient among the ribozymes that targeted different accessible sites of HCV IRES (data not shown).

The trans-splicing and trans-cleavage analyses indicated, therefore, that the relative ribozyme accessibility of the target sites corresponded with the predicted accessibility from the mapping analyses.

Comparison of ribozyme activities in vitro and in cells To compare the Rib199 ribozyme activity with other ribozymes, we incubated various ribozymes that targeted different sites in HCV IRES with the substrate RNA in vitro, amplified transsplicing products, and analyzed the relative ribozyme activity by assessing the relative amounts of the reaction products (Fig. 4A). Rib195, Rib196, Rib199, and Rib251, which targeted uridines in HCV IRES that were detected via the mapping analysis, efficiently employed the trans-splicing reaction with the target RNA. In contrast, Rib86, Rib329, and Rib380, which targeted uridines that were not identified from our mapping study, barely performed a trans-splicing reaction with the HCV IRES RNA. Noticeably, Rib199 that targeted the site that was predicted to be the most accessible by the mapping study harbored the highest ribozyme activity in vitro among all the ribozymes that were tested in this study.

Once it was determined that the ribozymes that specifically recognized the most accessible sites of the substrate efficiently reacted with the target RNA in vitro, we then determined if the
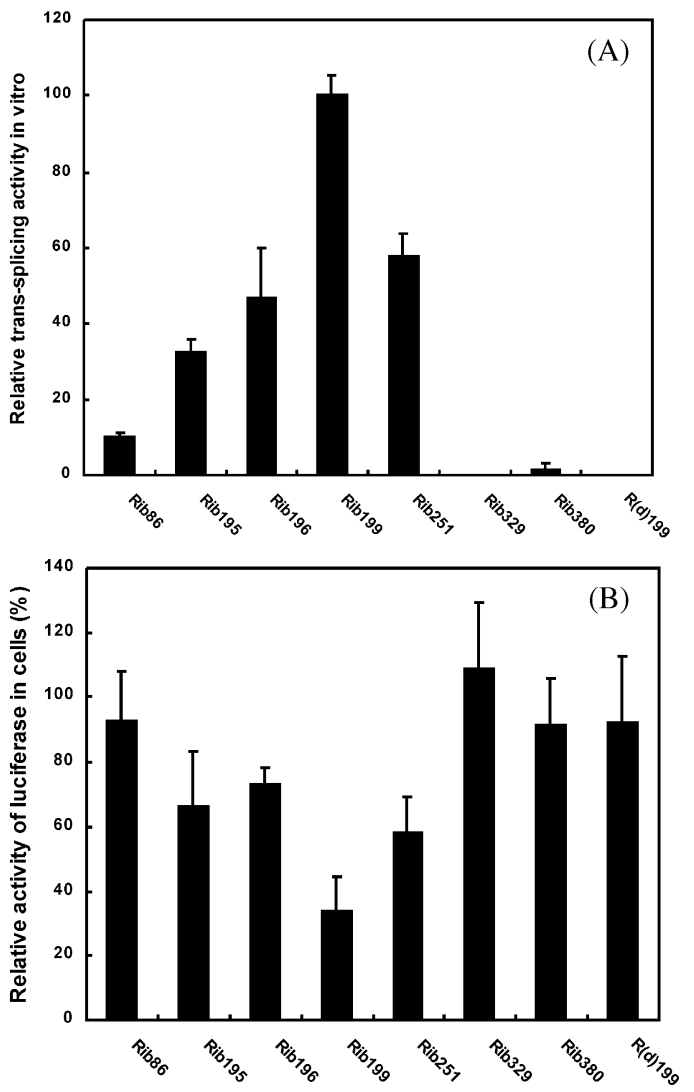

Fig. 4. Ribozyme activity in vitro and in cells. (A) Ribozyme trans-splicing activity in vitro. Specific ribozymes that recognize individual sites on HCV IRES or the inactive version of the ribozymes were incubated with HCV IRES. The trans-splicing reaction products were amplified and analyzed, described in Fig. 3A. The PCR products were quantified using Alphaimager ${ }^{\mathrm{TM}}$ 2200 (Alpha Innotech). The relative trans-splicing activity of each ribozyme was represented as the trans-splicing products that were normalized with the amount of PCR products of the reaction with Rib199. (B) Ribozyme cleavage activity in cells. The 293T cells were co-transfected with the control tRNA, specific ribozymes, or inactive ribozymes along with IRES/FLuc RNA as a reporter RNA. The relative luciferase activity was quantitated as a percentage of the sample that was transfected with the control tRNA. The values represent the means plus and minus standard deviation of three separate measurements.

ribozymes could also be active in cells. To this effect, we assessed the intracellular trans-cleavage activity of the ribozymes by measuring and comparing the reporter firefly luciferase activity in the cells that were co-transfected with individual ribozyme and IRES/FLuc RNA that encoded the reporter firefly luciferase (FLuc) linked to HCV IRES (Fig. $4 \mathrm{~B})$. If the ribozymes were truly active in cells, then the intracellular cleavage by the ribozymes would result in a reduction in the target RNA amount, thereby reducing the luciferase activity. To control and normalize for differences in the HCV IRES-dependent FLuc expression that is due to transfection efficiency, we also co-transfected RNA that 
encodes renilla luciferase (RLuc). Rib195, Rib196, Rib199, and Rib251 reduced the HCV IRES-dependent expression of the reporter gene; whereas, the inactive version of the ribozyme, R(d)199, showed little inhibition. Rib199 in particular blocked the IRES-dependent expression of the reporter gene the most effectively and reduced the expression by $70 \%$ relative to the control tRNA. Furthermore, Rib86, Rib329, and Rib380, which were poorly reactive with HCV IRES in vitro (as shown in Fig. 4A), also barely inhibited the expression of IRES/FLuc RNA. Thus, the reduction of the IRES/FLuc expression by Rib195, Rib196, Rib199, and Rib251 could be mainly due to the intracellular cleavage of HCV IRES RNA. These results also strongly indicated that the relative ribozyme-accessibility of the target sites in the cells corresponded with the accessibility which were predicted by mapping and in vitro analyses.

In this study, by the use of mapping studies, we identified the loop IIIb regions, especially uridine at position 199, in HCV IRES as the most accessible to ribozymes. Moreover, the trans-splicing and trans-cleavage analyses in vitro, and the trans-cleavage reaction in cells clearly showed that the ribozymes that recognize these identified accessible sites are truly most active. Other groups also demonstrated that hammerhead ribozymes that targeted the loop IIIb of HCV IRES were more effective than others (Macejak et al., 2000). However, they chose ribozymes randomly, not by rational mapping studies, and also did not analyze the activity of ribozyme that recognized uridine at position 199. Recently, many anti-viral protocols that are based on inhibitory RNA or short oligonucleotides (such as trans-cleavage ribozyme, trans-splicing ribozyme, antisense oligonucleotides, or siRNA) have been proposed (Sullenger and Gilboa, 2002). One key factor that influences the efficiency of this RNAbased viral suppression is the accessibility of the substrate RNA for the inhibitory RNA binding. Therefore, mapping studies, as performed here, could be generally used in a wide range of studies for optimizing the intracellular anti-HCV activity with various inhibitory RNAs. Furthermore, the mapping analyses that were developed in this study could be potentially exploited to isolate the most accessible sites, also in other viral RNAs or tumor-associated unique RNAs, for the therapeutic development of other infectious or malignant diseases.

Acknowledgments We are deeply thankful to S. K. Jang (POSTECH University) for his generous gift of the $\mathrm{pH}(18-$ 402)CAT vector and $\mathrm{pR} / \mathrm{HCV} / \mathrm{F}$, and B. Sullenger (Duke University) for pT7L-21. The present research was conducted by the research fund of Dankook University in 2001.

\section{References}

Been, M. and Cech, T. (1986) One binding site determines sequence specificity of Tetrahymena pre-rRNA self-splicing, trans-splicing, and RNA enzyme activity. Cell 47, 207-216.

Bukh, J., Purcell, R. H. and Miller, R. H. (1992) Sequence analysis of 5' noncoding region of hepatitis $\mathrm{C}$ virus. Proc. Natl. Acad. Sci. USA 89, 4942-4946.

Choo, Q. -L. Richman, K. H., Han, J. H., Berger, K., Lee, C., Dong, C., Gallegos, C., Coit, D., Medina-Selby, A., Barr, P. J., Weiner, A. J., Bradley, D. W., Kuo, G. and Houghton, M. (1991) Genetic organization and diversity of the hepatitis C virus. Proc. Natl. Acad. Sci. USA 88, 2451-2455.

Chung, J. H., Park, H. -Y., Lee, J. H. and Jang, Y. (2002) Identification of the dITP- and XTP-hydrolyzing protein from Escherichia coli. J. Biochem. Mol. Biol. 35, 403-408.

Di Bisceglie, A. M., McHutchison, J. F. and Rice, C. M. (2002) New therapeutic strategies for hepatitis C. Hepatology 35, 224231.

Fried, M. W., Shiffman, M. L., Reddy, K. R., Smith, C., Marinos, G., Goncales, F. L. Jr., Haussinger, D., Diago, M., Carosi, G., Dhumeaux, D., Craxi, A., Lin, A., Hoffman, J. and Yu, J. (2002) Peginterferon alfa-2a plus ribavirin for chronic hepatitis C virus infection. N. Engl. J. Med. 347, 975-982.

Hahm, B., Kim, Y. K., Kim, J. H., Kim, T. Y. and Jang, S. K. (1998) Heterogeneous nuclear ribonucleoprotein L interacts with the 3' border of the internal ribosomal entry site of hepatitis C virus. J. Virol. 72, 8782-8788.

Hanecak, R., Browndriver, V., Fox, M. C., Azad, R. F., Furusako, S., Nozaki, C., Ford, C., Sasmor, H. and Anderson, K. P. (1996) Antisense oligonucleotide inhibition of hepatitis C virus gene expression in transformed hepatocytes. J. Virol. 70, 52035212.

Honda, M., Beard, M. R., Ping, L. H. and Lemon, S. M. (1999) A phylogenetically conserved stem-loop structure at the $5^{\prime}$ border of the internal ribosome entry site of hepatitis $\mathrm{C}$ virus is required for cap-independent viral translation. J. Virol. 73, $1165-1174$.

Jones, J. T., Lee, S. -W. and Sullenger, B. A. (1996) Tagging ribozyme reaction sites to follow trans-splicing in mammalian cells. Nat. Med. 2, 643-648.

Jones, J. T. and Sullenger, B. A. (1997) Evaluating and enhancing ribozyme reaction efficiency in mammalian cells. Nat. Biotechnol. 15, 902-905.

Kapadia, S. B., Brideau-Anderson, A. and Chisari, F. V. (2003) Interference of hepatitis $\mathrm{C}$ virus RNA replication by short interfering RNAs. Proc. Natl. Acad. Sci. USA 100, 2014-2018.

Kim, H. S. and Park, Y. S. (2002) Effect of lipid compositions on gene transfer into 293 cells using sendai $\mathrm{F} / \mathrm{HN}$-virosomes. $J$. Biochem. Mol. Biol. 35, 459-464.

Lan, N., Howrey, R. P., Lee, S. -W., Smith, C. A. and Sullenger, B. A. (1998) Ribozyme-mediated repair of sickle $\beta$-globin mRNAs in erythrocyte precursors. Science 280, 1593-1596.

Lan, N., Rooney, B. L., Lee, S. -W., Howrey, R. P., Smith, C. A. and Sullenger, B. A. (2000) Enhancing RNA repair efficiency by combining trans-splicing ribozymes that recognize different accessible sites on a target RNA. Mol. Ther. 2, 245-255.

Lauer, G. M. and Waker, B. D. (2001) Hepatitis C virus infection. N. Engl. J. Med. 345, 41-52.

Lieber, A., He, C. Y., Polyak, S. J., Gretch, D. R., Barr, D. and Kay, M. A. (1996) Elimination of hepatitis C virus RNA in infected human hepatocytes by adenovirus-mediated expression of ribozymes. J. Virol. 70, 8782-8791.

Macejak, D. G., Jensen, K. L., Jamison, S. F., Domenico, K., 
Roberts, E. C., Chaudhary, N., von Carlowitz, I., Bellon, L., Tong, M. J., Conrad, A., Pavco, P. A. and Blatt, L. M. (2000) Inhibition of hepatitis $\mathrm{C}$ virus (HCV)-RNA-dependent translation and replication of a chimeric HCV poliovirus using synthetic stabilized ribozymes. Hepatology 31, 769-776.

Manns, M. P., McHutchison, J. G., Gordon, S. C., Rustgi, V. K., Shiffman, M., Reindollar, R., Goodman, Z. D., Koury, K., Ling, M. and Albrecht, J. K. (2001) Peginterferon alfa-2b plus ribavirin compared with interferon alfa- $2 b$ plus ribavirin for initial treatment of chronic hepatitis $\mathrm{C}$ : a randomised trial. Lancet 358, 958-965.

Miller, R. H. and Purcell, R. H. (1990) Hepatitis C virus shares amino acid sequence similarity with pestiviruses and flaviviruses as well as members of two plant virus supergroups. Proc. Natl. Acad. Sci. USA 87, 2057-2061.

Phylactou, L. A., Darrah, C. and Wood, M. J. (1998) Ribozymemediated trans-splicing of a trinucleotide repeat. Nat. Genet. 18, 378-381.

Randall, G., Grakoul, A, and Rice, C. M. (2003) Clearance of replicating hepatitis $\mathrm{C}$ virus replicon RNAs in cell culture by small interfering RNAs. Proc. Natl. Acad. Sci. USA 100, 235240.

Rogers, C. S., Vanoye, C. G., Sullenger, B. A. and George, Jr., A. L. (2002) Functional repair of a mutant chloride channel using a trans-splicing ribozyme. J. Clin. Invest. 110, 1783-1798.

Rosenberg, S. (2001) Recent advances in the molecular biology of hepatitis C virus. J. Mol. Biol. 313, 451-464.

Ryu, K. -J., Kim, J. -H. and Lee, S. -W. (2003) Ribozymemediated selective induction of new gene activity in hepatitis $\mathrm{C}$ virus internal ribosome entry site-expressing cells by targeted trans-splicing. Mol. Ther. 7, 386-395.

Sakamoto, N., Wu, C. H. and Wu, G. Y. (1996) Intracellular cleavage of hepatitis $\mathrm{C}$ virus RNA and inhibition of viral protein translation by hammerhead ribozymes. J. Clin. Invest. 98, 2720-2728
Shin, K. -S., Bae, S. -J., Hwang, E. -S., Jeong, S. and Lee, S. -W. (2002) Ribozyme-mediated replacement of p53 RNA by targeted trans-splicing. J. Microbiol. Biotechnol. 12, 844-848.

Sullenger, B. A. (1996) Ribozyme-mediated repair of RNAs encoding mutant tumor suppressors. Cytokines Mol. Ther. 2, 201-206.

Sullenger, B. A. and Cech, T. R. (1994) Ribozyme-mediated repair of defective mRNA by targeted trans-splicing. Nature 317, 619-622.

Sullenger, B. A. and Gilboa, E. (2002). Emerging clinical applications of RNA. Nature 418, 252-258.

Wang, T. H., Rijnbrand, R. C. and Lemon, S. M. (2000) Core protein-coding sequence, but not core protein, modulates the efficiency of cap-independent translation directed by the internal ribosome entry site of hepatitis C virus. J. Virol. 74, 11347-11358.

Watanabe, T. and Sullenger, B. A. (2000) Induction of wild-type p53 activity in human cancer cells by ribozymes that repair mutant p53 transcripts. Proc. Natl. Acad. Sci. USA 97, 84908494.

Welch, P. J., Tritz, R., Yei, S., Leavitt, M., Yu, M. and Barber, J. (1996) A potential therapeutic application of hairpin ribozymes: in vitro and in vivo studies of gene therapy for hepatitis $\mathrm{C}$ virus infection. Gene Ther. 3, 994-1001.

Wilson, J. A., Jayasena, S., Khvorova, A., Sabatinos, S., RodrigueGervais, I. G., Arya, S., Sarangi, F., Harris-Brandts, M., Beaulieu, S. and Richardson, C. D. (2003) RNA interference blocks gene expression and RNA synthesis from hepatitis C replicons propagated in human liver cells. Proc. Natl. Acad. Sci. USA 100, 2783-2788.

Witherell, G. W. (2001) ISIS-14803 (ISIS Pharmaceuticals). Curr. Opin. Investig. Drugs. 2, 1523-1529.

World Health Organization. (1999) Global surveillance and control of hepatitis C. J Viral Hepat. 6, 35-47. 